Research Article

\title{
Study on Force Schemes in Pseudopotential Lattice Boltzmann Model for Two-Phase Flows
}

\author{
Yong Peng $\mathbb{D}$, Bo Wang $\mathbb{D}$, and Yunfei Mao \\ State Key Laboratory of Hydraulics and Mountain River Engineering, Sichuan University, Chengdu, Sichuan 610065, China \\ Correspondence should be addressed to Bo Wang; wangbo@scu.edu.cn
}

Received 7 July 2017; Revised 11 October 2017; Accepted 22 November 2017; Published 9 January 2018

Academic Editor: Manfred Krafczyk

Copyright (C) 2018 Yong Peng et al. This is an open access article distributed under the Creative Commons Attribution License, which permits unrestricted use, distribution, and reproduction in any medium, provided the original work is properly cited.

\begin{abstract}
Multiphase flows are very important in industrial application. In present study, the force schemes in the pseudopotential LBM for two-phase flows have been compared in detail and the force schemes include Shan-Chen, EDM, MED, and Guo's schemes. Numerical simulations confirm that all four schemes are consistent with the Laplace law. For Shan-Chen scheme, the smaller $\tau$ is, the smaller the surface tension is. However, for other schemes, $\tau$ has no effect on surface tension. When $0.6<\tau \leq 1$, the achieved density ratio will reduce as $\tau$ reduces. During this range of $\tau$, the maximum density ratio of EDM scheme will be greater than that of other schemes. For a constant $T$, the curves of the maximum spurious currents $\left(u^{\prime}\right)$ has a minimum value which is corresponding to $\tau^{\prime}$ except for EDM schemes. In the region of $\tau^{\prime}<\tau \leq 1, u^{\prime}$ will reduce as $\tau$ decreases. On the other hand, in the area of $\tau \leq \tau^{\prime}$, $u^{\prime}$ will increase as $\tau$ decreases. However, for EDM scheme, $u^{\prime}$ will increase as $\tau$ increases.
\end{abstract}

\section{Introduction}

Multiphase flows are very important in industrial application [1]. Recently, the lattice Boltzmann method (LBM) has been applied widely for studying the two-phase flows and demonstrated its advantages [2-4]. The LBM for two-phase flows can be divided into the following four kinds: pseudopotential model [5, 6], free energy model [7], kinetic theory based model [8], and color model $[9,10]$, in which the pseudopotential LBM is very popular because of its simplicity.

In pseudopotential LBM, the interactions between fluids are simulated by an artificial interparticle potential. So, the force scheme is very important to simulate two-phase flows accurately. There are four main kinds of force schemes in the pseudopotential LBM: the first is Shan and Chen's force scheme $[5,6]$, the second is the Exact Difference Method (EDM) scheme [11-13], the third is Method of Explicit Derivative (MED) scheme [8], and the last is Guo's scheme which considered the discrete lattice effects [14]. Shan [15] showed that the spurious current present in LBM is due to the insufficient isotropy of operator and proposed a method to improve it. Shan [16] proposed a general approach of calculating the pressure tensor for nonideal gas LBM. Yu and Fan [17] combined adaptive mesh refinement method and lattice Boltzmann method to improve the two-phase flow simulation. It should be noted that the EDM scheme is Galilean invariant and the results obtained by EDM scheme do not depend on relaxation time $[11,18]$. Kupershtokh et al. [13] compared three kinds of approximation of the gradient of special potential ("local," "mean-value," and "general") and showed that the "general" approximation was most precise and stable. Moreover, the maximal density ratio was larger than $10^{7}$ for the van der Waals Equation of State and up to $10^{9}$ for the Carnahan-Starling Equation of State. Recently, Huang et al. [19] and Sun et al. [20] investigated the performance of different forcing schemes in the pseudopotential LBM. Li et al. [21] carried out a theoretical analysis of the Shan-Chen and EDM schemes in the pseudopotential LBM. Hu et al. [22] carried out the comparative analysis of different force schemes in pseudopotential LBM. Zheng et al. [23] studied the different force treatments in detail in Shan-Chen twophase LBM by theoretical analysis.

Based on above analysis, although some studies have been carried out on the force schemes in pseudopotential LBM, but the detailed comparison of different force schemes is scarce in 
available literature. In present study, a detailed comparison of force schemes including Shan-Chen, EDM, MED, and Guo's schemes will be carried out.

\section{Pseudopotential Lattice Boltzmann Model for Two-Phase Flows}

2.1. Shan-Chen Scheme. The first pseudopotential lattice Boltzmann model for two-phase flows is proposed by Shan and Chen $[5,6]$ and is expressed as follows:

$$
\begin{aligned}
f_{\alpha}\left(\mathbf{x}+\mathbf{e}_{\alpha} \Delta t, t+\Delta t\right)= & f_{\alpha}(\mathbf{x}, t) \\
& -\frac{1}{\tau}\left[f_{\alpha}(\mathbf{x}, t)-f_{\alpha}^{\mathrm{eq}}(\rho, \mathbf{u})\right], \\
\mathbf{u}^{\mathrm{eq}}= & \mathbf{u}(\mathbf{x}, t)+\tau \Delta \mathbf{u}, \\
\Delta \mathbf{u}(\mathbf{x}, t)= & \frac{\mathbf{F}(\mathbf{x}, t) \Delta t}{\rho(\mathbf{x}, t)} .
\end{aligned}
$$
by

The equilibrium distribution functions can be calculated

$$
f_{\alpha}^{\text {eq }}(\rho, \mathbf{u})=\rho w_{\alpha}\left(1+\frac{\mathbf{e}_{\alpha} \mathbf{u}}{\theta}+\frac{\left(\mathbf{e}_{\alpha} \mathbf{u}\right)^{2}}{2 \theta^{2}}-\frac{\mathbf{u}^{2}}{2 \theta}\right)
$$

The density and flow velocity can be obtained by the following:

$$
\begin{aligned}
& \rho=\sum_{\alpha} f_{\alpha}, \\
& \mathbf{u}=\frac{1}{\rho} \sum_{\alpha} \mathbf{e}_{\alpha} f_{\alpha} .
\end{aligned}
$$

The actual fluid velocity can be defined according to Ginzburg and Adler [24] as

$$
\widetilde{\mathbf{u}}=\mathbf{u}+\frac{\mathbf{F} \Delta t}{(2 \rho)}
$$

2.2. EDM Scheme. The pseudopotential lattice Boltzmann model with EDM $[11,12]$ can read

$$
\begin{aligned}
f_{\alpha}\left(\mathbf{x}+\mathbf{e}_{\alpha} \Delta t, t+\Delta t\right)= & f_{\alpha}(\mathbf{x}, t) \\
& -\frac{1}{\tau}\left[f_{\alpha}(\mathbf{x}, t)-f_{\alpha}^{\mathrm{eq}}(\rho, \mathbf{u})\right] \\
& +f_{\alpha}^{\mathrm{eq}}(\rho, \mathbf{u}+\Delta \mathbf{u}) \\
& -f_{\alpha}^{\mathrm{eq}}(\rho, \mathbf{u})
\end{aligned}
$$

where $\rho \mathbf{u}=\sum_{\alpha} \mathbf{e}_{\alpha} f_{\alpha}, \Delta \mathbf{u}=\mathbf{F} \Delta t / \rho$ and the equilibrium distribution function is shown as follows:

$$
f_{\alpha}^{\mathrm{eq}}(\rho, \mathbf{u})=\rho w_{\alpha}\left(1+\frac{\mathbf{e}_{\alpha} \mathbf{u}}{\theta}+\frac{\left(\mathbf{e}_{\alpha} \mathbf{u}\right)^{2}}{2 \theta^{2}}-\frac{\mathbf{u}^{2}}{2 \theta}\right) .
$$

The real fluid velocity can be obtained from Ginzburg and Adler [24] as follows:

$$
\widetilde{\mathbf{u}}=\mathbf{u}+\frac{\mathbf{F} \Delta t}{2 \rho} .
$$

2.3. Guo's Scheme. The pseudopotential lattice Boltzmann model with Guo's force scheme [14] can be shown as follows:

$$
\begin{aligned}
f_{\alpha}\left(\mathbf{x}+\mathbf{e}_{\alpha} \Delta t, t+\Delta t\right)= & f_{\alpha}(\mathbf{x}, t) \\
& -\frac{1}{\tau}\left[f_{\alpha}(\mathbf{x}, t)-f_{\alpha}^{\mathrm{eq}}(\rho, \mathbf{u})\right] \\
& +\left(1-\frac{1}{2 \tau}\right) F_{\alpha}(t) \Delta t,
\end{aligned}
$$

with $F_{\alpha}=w_{\alpha}\left[\left(\mathbf{e}_{\alpha}-\widetilde{\mathbf{u}}\right) / C_{s}^{2}+\left(\mathbf{e}_{\alpha} \widetilde{\mathbf{u}} / C_{s}^{4}\right) \mathbf{e}_{\alpha}\right] \mathbf{F}$

The density and flow velocity can be obtained by the following [24]:

$$
\begin{aligned}
& \rho=\sum_{\alpha} f_{\alpha}, \\
& \widetilde{\mathbf{u}}=\frac{1}{\rho} \sum_{\alpha} \mathbf{e}_{\alpha}\left(f_{\alpha}+\frac{\Delta t}{2} F_{\alpha}\right) .
\end{aligned}
$$

2.4. MED Scheme. The pseudopotential lattice Boltzmann model with MED force scheme [8] can be shown as follows:

$$
\begin{aligned}
\bar{f}_{\alpha}(\mathbf{x} & \left.+\mathbf{e}_{\alpha} \Delta t, t+\Delta t\right)-\bar{f}_{\alpha}(\mathbf{x}, t) \\
= & -\frac{1}{\tau}\left[\bar{f}_{\alpha}(\mathbf{x}, t)-\bar{f}_{\alpha}^{\mathrm{eq}}(\rho, \mathbf{u})\right] \\
& +\Delta t\left(1-\frac{1}{2 \tau}\right) \frac{\left(\mathbf{e}_{\alpha}-\mathbf{u}\right) \cdot \mathbf{F}}{\rho c_{s}^{2}} f_{\alpha}^{\mathrm{eq}}(\rho, \mathbf{u}),
\end{aligned}
$$

with $\bar{f}_{\alpha}=f_{\alpha}-(\Delta \mathrm{t} / 2)\left(\left(\left(\mathbf{e}_{\alpha}-\mathbf{u}\right) \cdot \mathbf{F}\right) / \rho c_{s}^{2}\right) f_{\alpha}^{\mathrm{eq}}$.

The density and flow velocity can be obtained by

$$
\begin{aligned}
\rho & =\sum_{\alpha} \bar{f}_{\alpha}, \\
\rho \mathbf{u} & =\sum_{\alpha} \bar{f}_{\alpha} \mathbf{e}_{\alpha}+\frac{\Delta t}{2} \mathbf{F} .
\end{aligned}
$$

\section{Carnahan-Starling Equation of State (C-S EOS)}

In order to get relatively large density ratio, the CarnahanStarling Equation of State (C-S EOS) is used in the present study [25]:

$$
p=\rho R T \frac{1+b \rho / 4+(b \rho / 4)^{2}-(b \rho / 4)^{3}}{(1-b \rho / 4)^{3}}-a \rho^{2},
$$




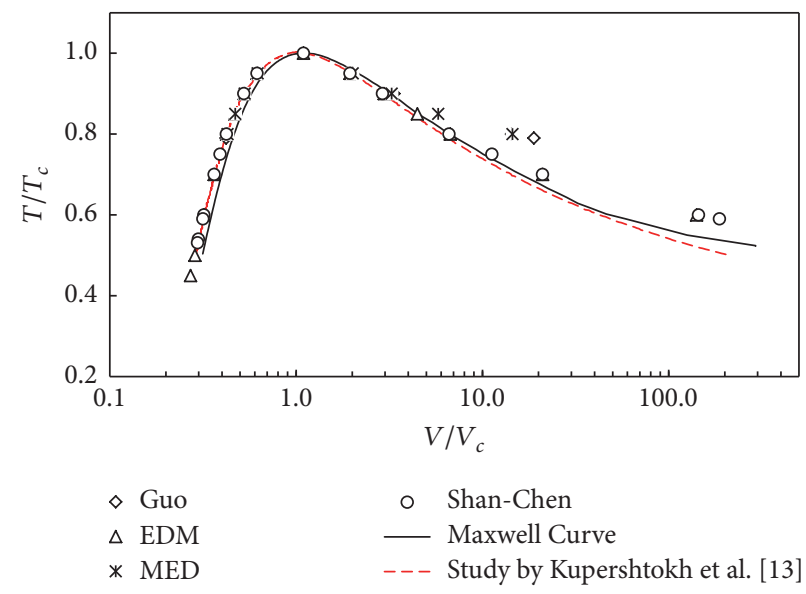

FIGURE 1: Predicted coexistence densities of two-phase separation by of Shan-Chen, EDM, MED, and Guo's schemes $(\tau=1)$.

in which $a=0.4963 R^{2} T_{c}^{2} / p_{c}, b=0.18727 R T_{c} / p_{c}$, and $T_{c}$ and $P_{c}$ can be obtained by

$$
\begin{aligned}
& T_{c}=\frac{0.3773 a}{b R}, \\
& p_{c}=\frac{0.0706}{b^{2}},
\end{aligned}
$$

where $a=1, b=4, R=1$ are used in the present study.

\section{Application Cases}

4.1. Two-Phase Separation. In this section, two-phase separation will be used to test four schemes of Shan-Chen, EDM, MED, and Guo. The force term can be calculated by the following:

$$
\mathbf{F}(x, t)=-G \psi \sum_{\alpha} w_{\alpha} \psi\left(x+\mathbf{e}_{\alpha} \Delta t\right) \mathbf{e}_{\alpha}
$$

where $w_{\alpha}$ is weights and, for D2Q9 model, $w_{\alpha}=4 / 9(\alpha=1)$, $w_{\alpha}=1 / 9(\alpha=2,3,4,5), w_{\alpha}=1 / 36(\alpha=6,7,8,9)$.

In the present work, the interaction potential is defined according to the method by Chen et al. [26] as follows:

$$
\psi(\rho)=\sqrt{\frac{2\left(p-\rho c_{s}^{2}\right)}{G c_{s}^{2}} .}
$$

In computation, $\rho_{0}=1,101 \times 101$ grids have been used; the periodic boundary conditions are used for all boundaries. The initial density for whole area is critical density, and a random density fluctuation of plus or minus $1 \%$ is added at the beginning for whole area. When the computation gets to equilibrium, gas and liquid phases will separate clearly. The simulated results are shown in Figure 1.

It can be known from Figure 1 that all of four schemes perform almost the same and agree well with the analytical solution. Besides, it is clear that the present study agrees with that by Kupershtokh et al. [13].

4.2. Surface Tension. Surface tension is of great importance in the two-phase flows and its relationship with bubble radius is consistent with the Laplace law. In this section, Laplace's law will be used to verify four schemes and the effect of $\tau$ and $T$ on the surface tension will be discussed. The simulated results are shown in Table 1 and Figures 2-4.

It can be seen from Figures 2 and 3, the pressure difference between the inside and outside the bubble is linearly related to the reciprocal of the radius. This agrees well with the Laplace's law. Figure 3 shows that $\tau$ has no effect on surface tension for the EDM, MED, and Guo's schemes.

4.3. Maximum Two-Phase Density Ratio. The study shows that the simulation will become unstable when $T$ is low for four schemes, so $T$ has a certain range for stable computation. To demonstrate the numerical stability of four schemes and get the minimum $T$ with stable computation, two-phase separation case is tested by adjusting $\tau$ and the results are shown in Figure 5.

It can be seen from Figure 5 that the minimum $T$ will become larger as $\tau$ reduces when $0.5<\tau \leq 1$ and it means that achieved maximum ratio of two-phase densities will become smaller. For the same $\tau$, the maximum density ratio of EDM scheme will be larger than those of other schemes for $0.6<\tau \leq 1$.

4.4. Spurious Currents. The spurious current is one of the important criteria to evaluate pseudopotential models because it will lead to the computation instability. In order to study the effect of four force schemes on spurious current, a series of tests have been carried out. The maximum spurious currents for different $T$ and $\tau$ have been shown in Table 2 and Figure 6. It shows that the spurious current will increase as $T$ reduces with the same $\tau$ for all of four schemes. That is 
TABLE 1: The effect of $\tau$ and $T$ on the surface tension for $T=0.8 T c$.

\begin{tabular}{|c|c|c|}
\hline$\tau$ & $1 / r$ & $d p$ \\
\hline \multicolumn{3}{|c|}{ EDM scheme } \\
\hline \multirow{6}{*}{0.75} & $4.14 E-02$ & $3.13 E-04$ \\
\hline & $4.50 E-02$ & $3.42 E-04$ \\
\hline & $4.95 E-02$ & $3.77 E-04$ \\
\hline & $5.49 E-02$ & $4.19 E-04$ \\
\hline & $6.16 E-02$ & $4.71 E-04$ \\
\hline & $7.28 E-02$ & $5.61 E-04$ \\
\hline \multirow{6}{*}{0.8} & $4.14 E-02$ & $3.14 E-04$ \\
\hline & $4.50 E-02$ & $3.42 E-04$ \\
\hline & $4.95 E-02$ & $3.77 E-04$ \\
\hline & $5.49 E-02$ & $4.20 E-04$ \\
\hline & $6.16 E-02$ & $4.72 E-04$ \\
\hline & $7.28 E-02$ & $5.62 E-04$ \\
\hline \multirow{6}{*}{0.85} & $4.14 E-02$ & $3.14 E-04$ \\
\hline & $4.50 E-02$ & $3.43 E-04$ \\
\hline & $4.95 E-02$ & $3.77 E-04$ \\
\hline & $5.49 E-02$ & $4.20 E-04$ \\
\hline & $6.16 E-02$ & $4.72 E-04$ \\
\hline & $7.28 E-02$ & $5.62 E-04$ \\
\hline \multirow{6}{*}{0.9} & $4.14 E-02$ & $3.14 E-04$ \\
\hline & $4.50 E-02$ & $3.43 E-04$ \\
\hline & $4.95 E-02$ & $3.78 E-04$ \\
\hline & $5.49 E-02$ & $4.20 E-04$ \\
\hline & $6.16 E-02$ & $4.72 E-04$ \\
\hline & $7.28 E-02$ & $5.62 E-04$ \\
\hline \multirow{6}{*}{0.95} & $4.14 E-02$ & $3.14 E-04$ \\
\hline & $4.50 E-02$ & $3.43 E-04$ \\
\hline & $4.95 E-02$ & $3.78 E-04$ \\
\hline & $5.49 E-02$ & $4.20 E-04$ \\
\hline & $6.16 E-02$ & $4.73 E-04$ \\
\hline & $7.28 E-02$ & $5.63 E-04$ \\
\hline \multirow{6}{*}{1} & $4.14 E-02$ & $3.14 E-04$ \\
\hline & $4.50 E-02$ & $3.43 E-04$ \\
\hline & $4.95 E-02$ & $3.78 E-04$ \\
\hline & $5.49 E-02$ & $4.21 E-04$ \\
\hline & $6.10 E-02$ & $4.71 E-04$ \\
\hline & $7.95 E-02$ & $6.15 E-04$ \\
\hline \multicolumn{3}{|c|}{ MED scheme } \\
\hline \multirow{6}{*}{0.8} & $4.19 E-02$ & $1.83 E-04$ \\
\hline & $4.58 E-02$ & $1.99 E-04$ \\
\hline & $5.05 E-02$ & $2.19 E-04$ \\
\hline & $5.64 E-02$ & $2.43 E-04$ \\
\hline & $6.38 E-02$ & $2.74 E-04$ \\
\hline & $7.38 E-02$ & $3.13 E-04$ \\
\hline \multirow{6}{*}{0.85} & $4.19 E-02$ & $1.83 E-04$ \\
\hline & $4.58 E-02$ & $1.99 E-04$ \\
\hline & $5.05 E-02$ & $2.19 E-04$ \\
\hline & $5.63 E-02$ & $2.43 E-04$ \\
\hline & $6.38 E-02$ & $2.73 E-04$ \\
\hline & $7.38 E-02$ & $3.13 E-04$ \\
\hline
\end{tabular}

TABLE 1: Continued.

\begin{tabular}{|c|c|c|}
\hline$\tau$ & $1 / r$ & $d p$ \\
\hline \multirow{6}{*}{0.9} & $4.19 E-02$ & $1.83 E-04$ \\
\hline & $4.58 E-02$ & $1.99 E-04$ \\
\hline & $5.05 E-02$ & $2.19 E-04$ \\
\hline & $5.63 E-02$ & $2.43 E-04$ \\
\hline & $6.38 E-02$ & $2.73 E-04$ \\
\hline & $7.38 E-02$ & $3.13 E-04$ \\
\hline \multirow{6}{*}{0.95} & $4.19 E-02$ & $1.83 E-04$ \\
\hline & $4.58 E-02$ & $1.99 E-04$ \\
\hline & $5.05 E-02$ & $2.19 E-04$ \\
\hline & $5.63 E-02$ & $2.43 E-04$ \\
\hline & $6.38 E-02$ & $2.73 E-04$ \\
\hline & $7.39 E-02$ & $3.13 E-04$ \\
\hline \multirow{6}{*}{1} & $4.19 E-02$ & $1.83 E-04$ \\
\hline & $4.58 E-02$ & $1.99 E-04$ \\
\hline & $5.05 E-02$ & $2.19 E-04$ \\
\hline & $5.63 E-02$ & $2.43 E-04$ \\
\hline & $6.38 E-02$ & $2.74 E-04$ \\
\hline & $7.39 E-02$ & $3.14 E-04$ \\
\hline \multicolumn{3}{|c|}{ Guo's scheme } \\
\hline \multirow{5}{*}{0.85} & $4.14 E-02$ & $1.97 E-04$ \\
\hline & $4.50 E-02$ & $2.16 E-04$ \\
\hline & $4.95 E-02$ & $2.38 E-04$ \\
\hline & $5.47 E-02$ & $2.65 E-04$ \\
\hline & $6.48 E-02$ & $3.17 E-04$ \\
\hline \multirow{6}{*}{0.9} & $4.14 E-02$ & $1.97 E-04$ \\
\hline & $4.50 E-02$ & $2.16 E-04$ \\
\hline & $4.95 E-02$ & $2.38 E-04$ \\
\hline & $5.47 E-02$ & $2.65 E-04$ \\
\hline & $6.48 E-02$ & $3.17 E-04$ \\
\hline & $7.54 E-02$ & $3.75 E-04$ \\
\hline \multirow{6}{*}{0.95} & $4.14 E-02$ & $1.97 E-04$ \\
\hline & $4.50 E-02$ & $2.16 E-04$ \\
\hline & $4.95 E-02$ & $2.38 E-04$ \\
\hline & $5.47 E-02$ & $2.65 E-04$ \\
\hline & $6.48 E-02$ & $3.17 E-04$ \\
\hline & $7.54 E-02$ & $3.74 E-04$ \\
\hline \multirow{6}{*}{1} & $4.14 E-02$ & $1.97 E-04$ \\
\hline & $4.50 E-02$ & $2.16 E-04$ \\
\hline & $4.95 E-02$ & $2.38 E-04$ \\
\hline & $5.47 E-02$ & $2.65 E-04$ \\
\hline & $6.48 E-02$ & $3.16 E-04$ \\
\hline & $7.54 E-02$ & $3.74 E-04$ \\
\hline
\end{tabular}

why computation is instable when $T$ is small. The maximum spurious current of MED scheme is slightly larger than that of other schemes. 

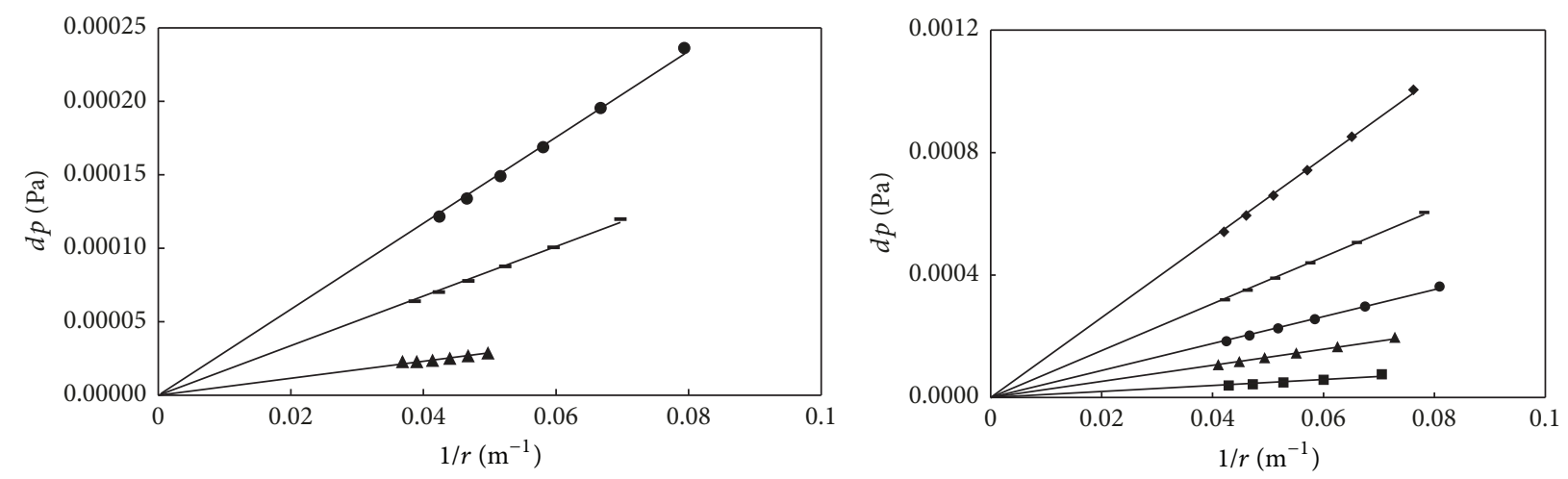

- $T / T c=0.86$

- $T / T c=0.9$

\ $T / T c=0.95$
- $T / T c=0.86$
- $T / T c=0.72$
- $T / T c=0.8$
- $T / T c=0.95$
\ $T / T c=0.9$

(a) Shan-Chen scheme

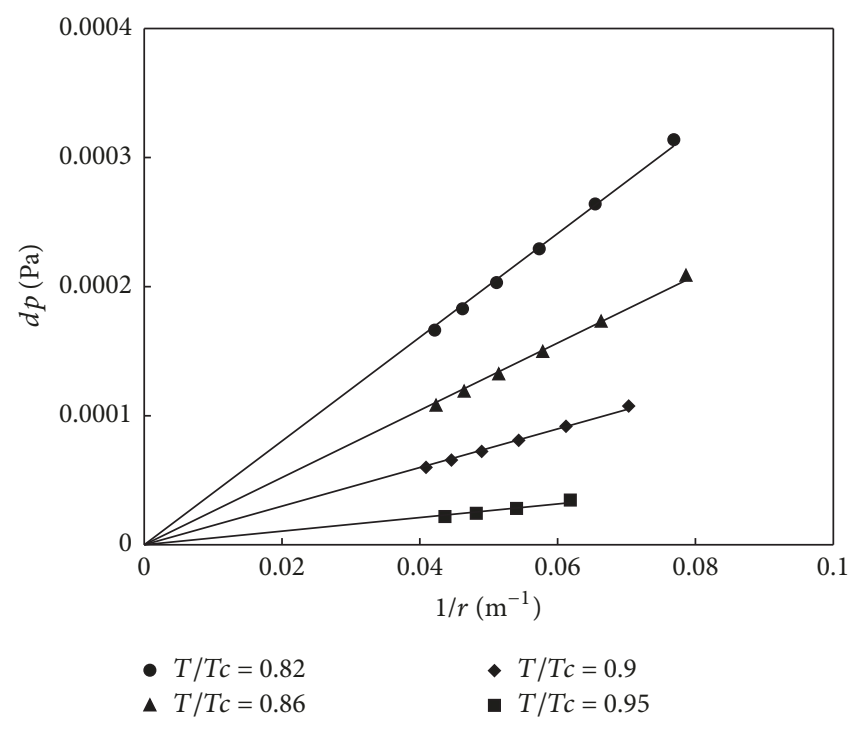

(c) Guo's scheme (b) EDM scheme

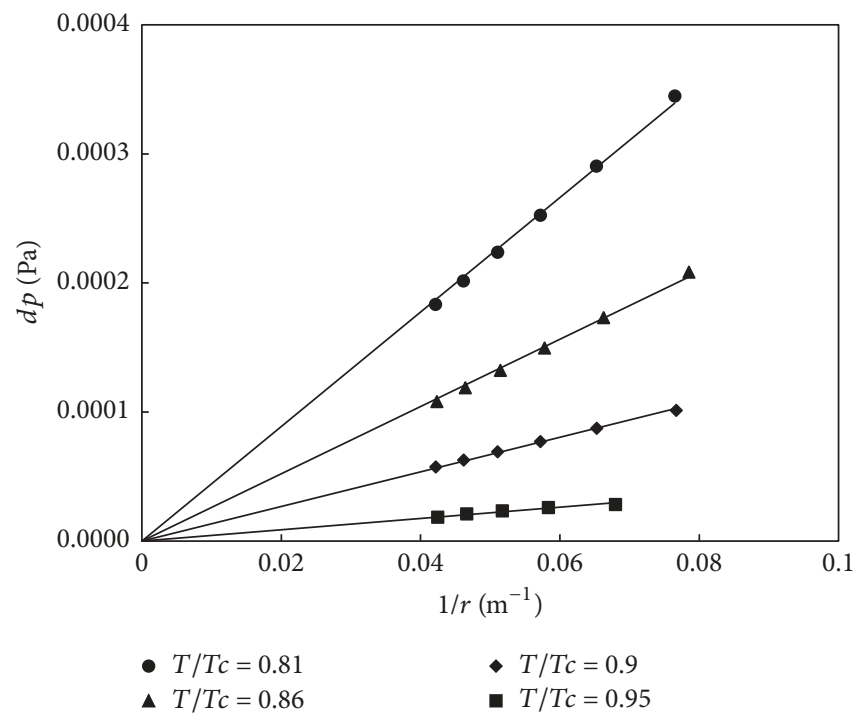

(d) MED scheme

FIGURE 2: Relationship between bubble radius and pressure difference at different temperatures for $\tau=0.7$.

Moreover, it can be seen from Figure 6 that, with a constant $T$, for Shan-Chen, MED, and Guo's schemes, the curves of $u^{\prime}$ have a minimum value which is corresponding to $\tau^{\prime}$. During this region of $\tau^{\prime}<\tau \leq 1, u^{\prime}$ will reduce as $\tau$ decreases. On the other hand, in the area of $\tau \leq \tau^{\prime}, u^{\prime}$ will increase as $\tau$ decreases. But for EDM scheme, $u^{\prime}$ will increase as $\tau$ increases. Moreover, present study is compared with that by Kupershtokh et al. [13] and both of results are similar as shown in Figure 7.

\section{Conclusions}

In present study, the force schemes in the pseudopotential LBM for two-phase flows have been compared in detail and the force schemes include Shan-Chen, EDM, MED, and Guo's schemes. The LBM with four schemes have been used to study the two-phase separation and surface tension. Besides, the maximum two-phase density ratio and spurious currents also are discussed in detail. Based on the above study, the following conclusions can be drawn:

(1) Numerical simulations confirm that all four schemes are consistent with the Laplace law. For the EDM, MED, and Guo's schemes, $\tau$ has no effect on surface tension.

(2) When $0.5<\tau \leq 1$, the achieved density ratio will reduce as $\tau$ reduces. For the same $\tau$, the maximum density ratio of EDM scheme will be greater than that of other schemes $0.6<\tau \leq 1$.

(3) Except for the EDM scheme, the curves of the maximum spurious currents $\left(u^{\prime}\right)$ have a minimum value which is corresponding to $\tau^{\prime}$ for three schemes. In the region of $\tau^{\prime}<\tau \leq 1, u^{\prime}$ will reduce as $\tau$ decreases. On the other hand, in the area of $\tau \leq \tau^{\prime}, u^{\prime}$ will increase as $\tau$ decreases. But for EDM scheme, $u^{\prime}$ will increase as $\tau$ increases. 


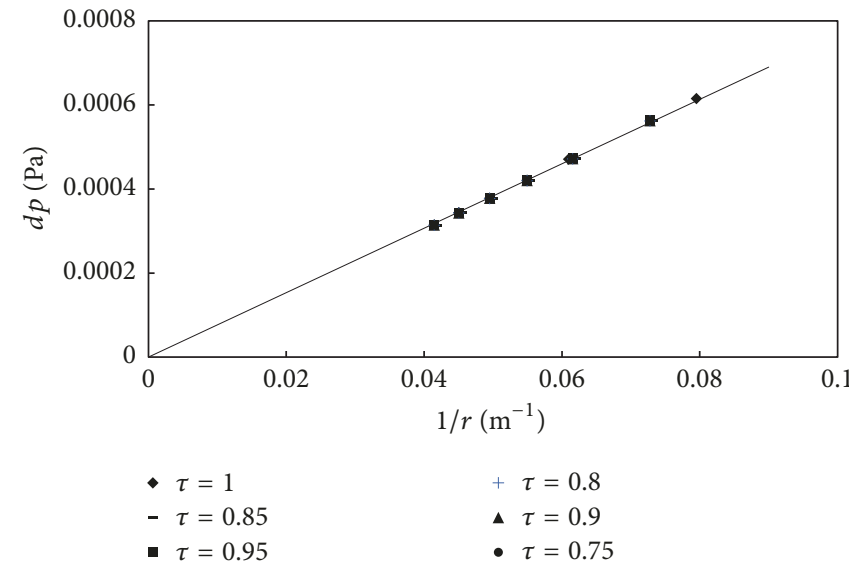

(a) EDM scheme

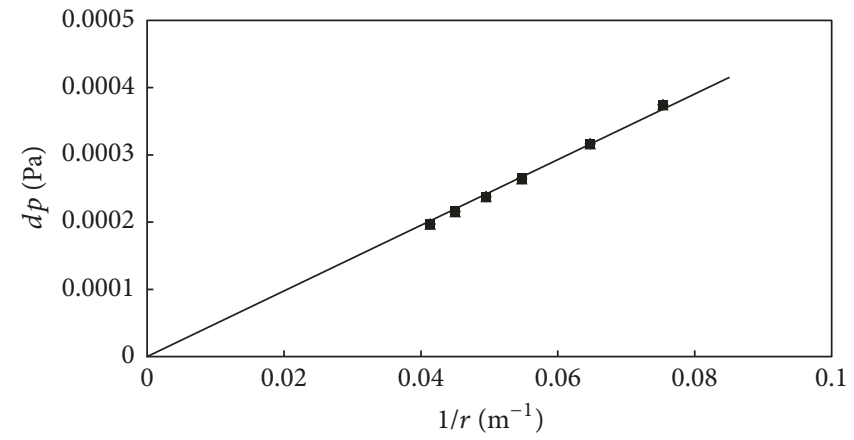

- $\tau=1$

- $\tau=0.95$

А $\tau=0.9$
- $\tau=0.85$

(b) Guo's scheme

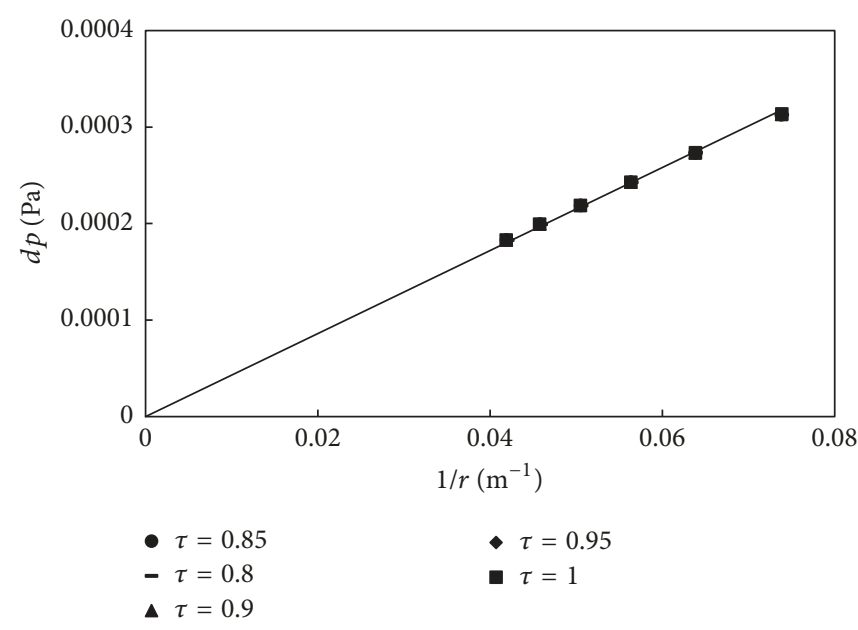

(c) MED scheme

FIGURE 3: Relationship between bubble radius and pressure difference at different $\tau$ for $T=0.8 T c$.

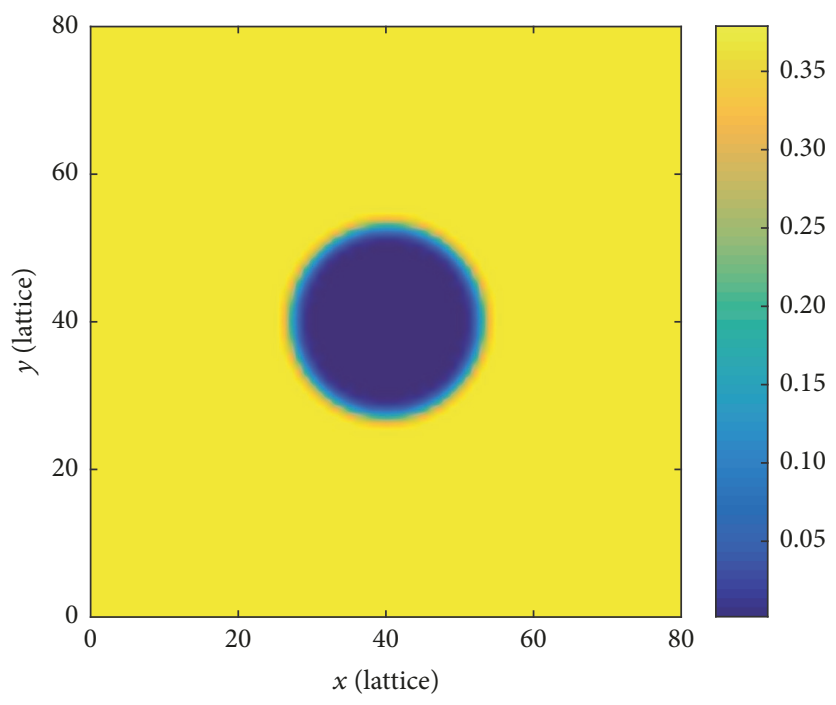

FIgURE 4: The density distribution of stable bubble with EDM force scheme. 


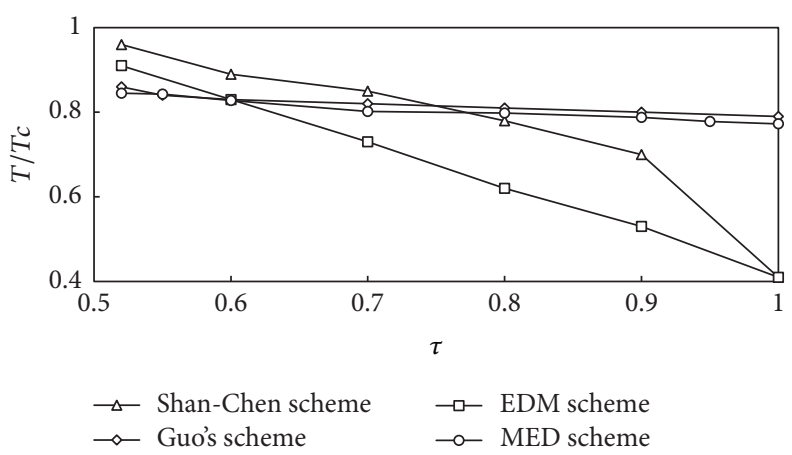

Figure 5: The minimum $T$ with stable computation at different $\tau$ for Shan-Chen, EDM, MED, and Guo's schemes.

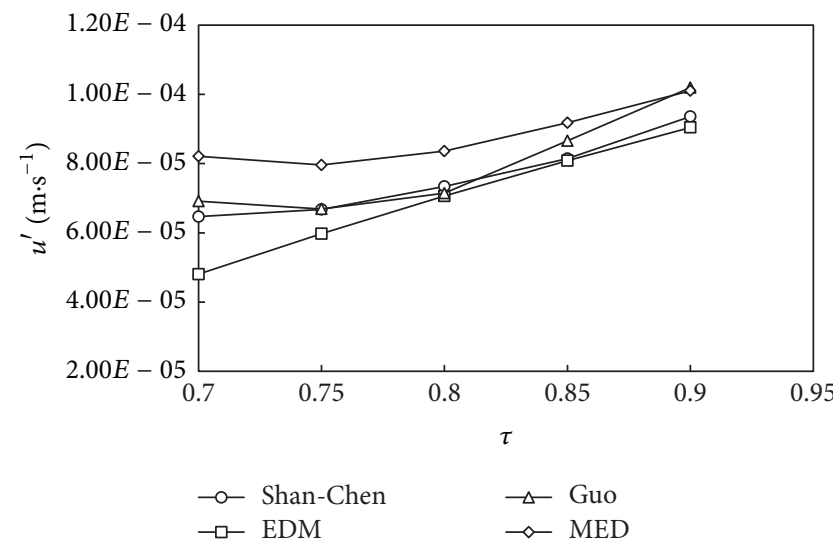

(a) $T=0.95 T c$

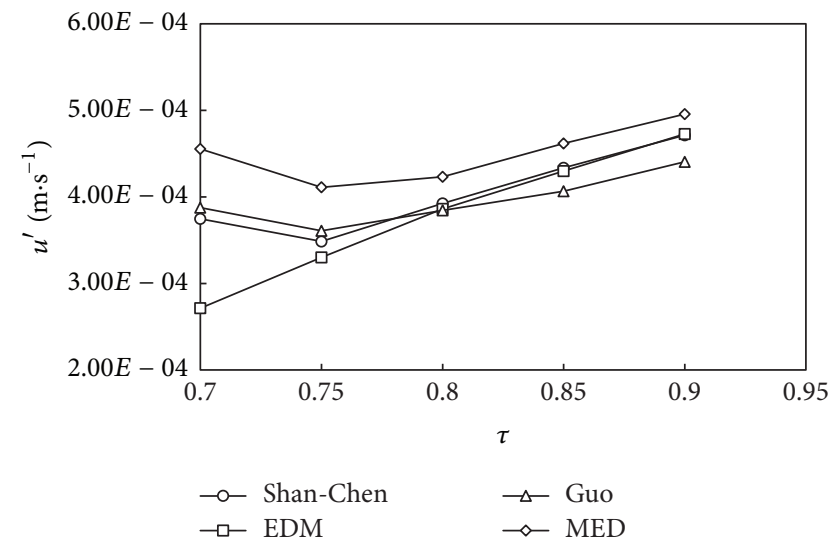

(b) $T=0.9 T c$

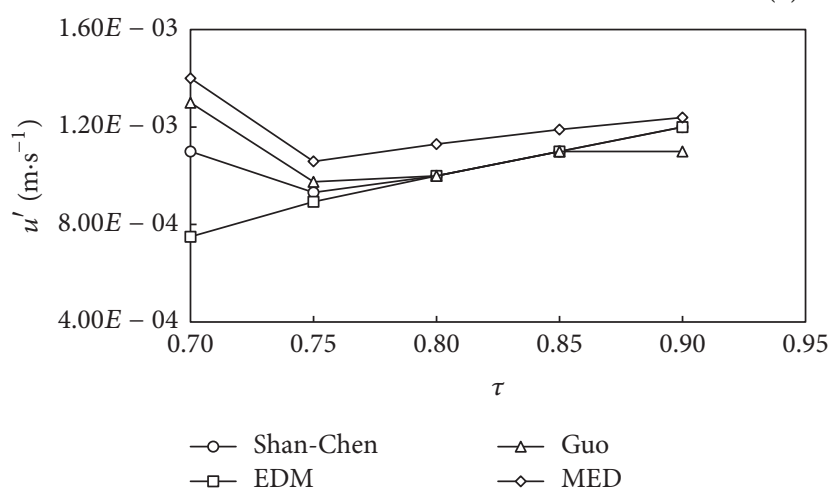

(c) $T=0.85 T c$

Figure 6: The maximum spurious currents of Shan-Chen, EDM, MED, and Guo's schemes for different $\tau$ and $T$.

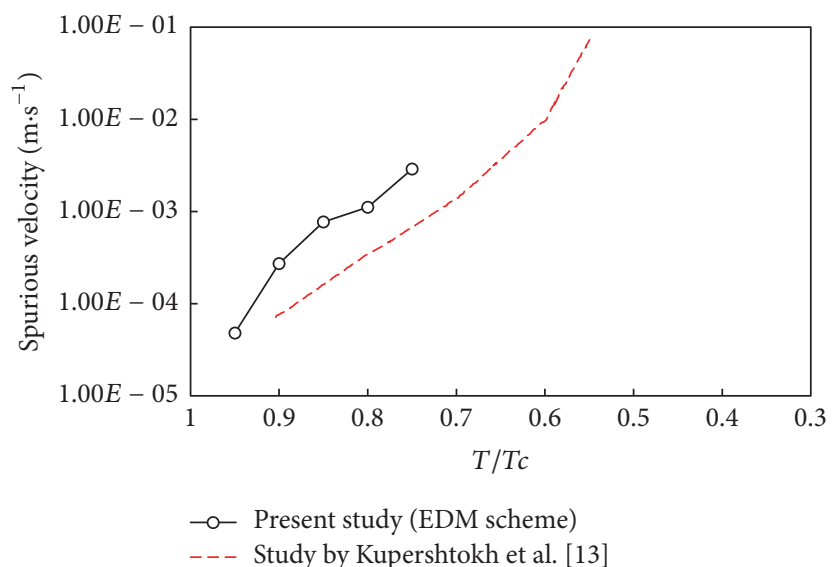

FIGURE 7: Comparison of maximum spurious currents for EDM scheme between Kupershtokh et al. [13] ( $\tau$ is unknown) and present study with $\tau=0.7$. 
TABLE 2: Maximum spurious currents for Shan-Chen, EDM, Guo's, and MED schemes.

\begin{tabular}{|c|c|c|c|c|c|}
\hline \multirow{2}{*}{$T / T c$} & \multirow{2}{*}{$\tau$} & \multicolumn{4}{|c|}{$u^{\prime}$} \\
\hline & & Shan-Chen scheme & EDM scheme & Guo's scheme & MED scheme \\
\hline \multirow{5}{*}{0.95} & 0.90 & $9.36 E-05$ & $9.04 E-05$ & $1.02 E-04$ & $1.01 E-04$ \\
\hline & 0.85 & $8.14 E-05$ & $8.09 E-05$ & $8.66 E-05$ & $9.18 E-05$ \\
\hline & 0.80 & $7.34 E-05$ & $7.07 E-05$ & $7.14 E-05$ & $8.36 E-05$ \\
\hline & 0.75 & $6.67 E-05$ & $5.97 E-05$ & $6.69 E-05$ & $7.96 E-05$ \\
\hline & 0.70 & $6.47 E-05$ & $4.81 E-05$ & $6.92 E-05$ & $8.21 E-05$ \\
\hline \multirow{5}{*}{0.90} & 0.90 & $4.71 E-04$ & $4.73 E-04$ & $4.41 E-04$ & $4.96 E-04$ \\
\hline & 0.85 & $4.34 E-04$ & $4.30 E-04$ & $4.07 E-04$ & $4.62 E-04$ \\
\hline & 0.80 & $3.93 E-04$ & $3.86 E-04$ & $3.84 E-04$ & $4.23 E-04$ \\
\hline & 0.75 & $3.49 E-04$ & $3.30 E-04$ & $3.61 E-04$ & $4.11 E-04$ \\
\hline & 0.70 & $3.75 E-04$ & $2.71 E-04$ & $3.88 E-04$ & $4.55 E-04$ \\
\hline \multirow{6}{*}{0.85} & 0.90 & $1.20 E-03$ & $1.20 E-03$ & $1.10 E-03$ & $1.24 E-03$ \\
\hline & 0.85 & $1.10 E-03$ & $1.10 E-03$ & $1.10 E-03$ & $1.19 E-03$ \\
\hline & 0.80 & $1.00 E-03$ & $1.00 E-03$ & $1.00 E-03$ & $1.13 E-03$ \\
\hline & 0.75 & $9.31 E-04$ & $8.94 E-04$ & $9.75 E-04$ & $1.06 E-03$ \\
\hline & 0.70 & $1.10 E-03$ & $7.50 E-04$ & $1.30 E-03$ & $1.40 E-03$ \\
\hline & 0.65 & $1.90 E-03$ & $7.72 E-04$ & - & $2.31 E-03$ \\
\hline
\end{tabular}

\section{Conflicts of Interest}

The authors declare that there are no conflicts of interest regarding the publication of this paper.

\section{Acknowledgments}

The first author would like to acknowledge the financial support of the National Natural Science Foundation of China (Grants nos. 51409183, 51579166, and 51611130203). Besides, this work was also supported by the National Key Technologies R\&D Program of China (no. 2015BAD24B01).

\section{References}

[1] M. Ishii and T. Hibiki, Thermo-Fluid Dynamics of Two-Phase Flow, Springer, New York, NY, USA, 2007.

[2] M. C. Sukop and D. T. Thorne, Lattice Boltzmann Modeling: An Introduction for Geoscientists and Engineers, Springer, Heidelberg, Germany, 2006.

[3] C. K. Aidun and J. R. Clausen, "Lattice Boltzmann method for complex flow," Annual Review of Fluid Mechanics, vol. 42, no. 1, pp. 439-472, 2010.

[4] H. Safari, M. H. Rahimian, and M. Krafczyk, "Consistent simulation of droplet evaporation based on the phase-field multiphase lattice Boltzmann method," Physical Review E, vol. 90, no. 3, Article ID 033305, 2014.

[5] X. Shan and H. Chen, "Lattice Boltzmann model for simulating flows with multiple phases and components," Physical Review E, vol. 47, no. 3, pp. 1815-1820, 1993.

[6] X. Shan and H. Chen, "Simulation of nonideal gases and liquid-gas phase transitions by the lattice Boltzmann equation," Physical Review E, vol. 49, no. 4, pp. 2941-2948, 1994.

[7] M. R. Swift, W. R. Osborn, and J. M. Yeomans, "Lattice Boltzmann simulation of non-ideal fluids," Physical Review Letters, vol. 75, no. 5, pp. 830-833, 1995.
[8] X. Y. He, X. W. Shan, and G. D. Doolen, "Discrete Boltzmann equation model for nonideal gases," Physical Review E, vol. 57, no. 1, pp. R13-R16, 1998.

[9] A. K. Gunstensen, D. H. Rothman, S. Zaleski, and G. Zanetti, "Lattice Boltzmann model of immiscible fluids," Physical Review A: Atomic, Molecular and Optical Physics, vol. 43, no. 8, pp. 4320-4327, 1991.

[10] J. Tölke, S. Freudiger, and M. Krafczyk, "An adaptive scheme using hierarchical grids for lattice Boltzmann multi-phase flow simulations," Computers \& Fluids, vol. 35, no. 8-9, pp. 820-830, 2006.

[11] A. L. Kupershtokh, "New method of incorporating a body force term into the lattice Boltzmann equation," in Proceedings of the 5th International EHD Workshop, pp. 241-246, 2004.

[12] A. L. Kupershtokh, "Criterion of numerical instability of liquid state in LBE simulations," Computers and Mathematics with Applications, vol. 59, no. 7, pp. 2236-2245, 2010.

[13] A. L. Kupershtokh, D. A. Medvedev, and D. I. Karpov, "On equations of state in a lattice Boltzmann method," Computers and Mathematics with Applications, vol. 58, pp. 965-974, 2009.

[14] Z. Guo, C. Zheng, and B. Shi, "Discrete lattice effects on the forcing term in the lattice Boltzmann method," Physical Review E, vol. 65, Article ID 046308, 2002.

[15] X. Shan, "Analysis and reduction of the spurious current in a class of multiphase lattice Boltzmann models," Physical Review E, vol. 73, no. 2, Article ID 047701, 2006.

[16] X. Shan, "Pressure tensor calculation in a class of nonideal gas lattice Boltzmann models," Physical Review E, vol. 77, no. 2, Article ID 066702, 2008.

[17] Z. Yu and L.-S. Fan, "An interaction potential based lattice Boltzmann method with adaptive mesh refinement AMR for two-phase flow simulation," Journal of Computational Physics, vol. 228, no. 17, pp. 6456-6478, 2009.

[18] P. J. Dellar, "Lattice Boltzmann formulation for linear viscoelastic fluids using an abstract second stress," SIAM Journal on Scientific Computing, vol. 36, no. 6, pp. A2507-A2532, 2014. 
[19] H. Huang, M. Krafczyk, and X. Lu, "Forcing term in singlephase and Shan-Chen-type multiphase lattice Boltzmann models," Physical Review E: Statistical, Nonlinear, and Soft Matter Physics, vol. 84, no. 4, Article ID 046710, 2011.

[20] K. Sun, T. Wang, M. Jia, and G. Xiao, "Evaluation of force implementation in pseudopotential-based multiphase lattice Boltzmann models," Physica A, vol. 391, no. 15, pp. 3895-3907, 2012.

[21] Q. Li, K. H. Luo, and X. J. Li, "Forcing scheme in pseudopotential lattice Boltzmann model for multiphase flows," Physical Review E, vol. 86, no. 1, Article ID 016709, 2012.

[22] A. Hu, L. Li, and R. Uddin, "Force method in a pseudo-potential lattice Boltzmann model," Journal of Computational Physics, vol. 294, pp. 78-89, 2015.

[23] L. Zheng, Q. Zhai, and S. Zheng, "Analysis of force treatment in the pseudopotential lattice Boltzmann equation method," Physical Review E, vol. 95, no. 4, Article ID 043301, 2017.

[24] I. Ginzburg and P. M. Adler, "Boundary flow condition analysis for the three-dimensional lattice Boltzmann model," Journal de Physique II, vol. 4, no. 2, pp. 191-214, 1994.

[25] N. F. Carnahan and K. E. Starling, "Intermolecular repulsions and the equation of state for fluids," AIChE Journal, vol. 18, no. 6, pp. 1184-1189, 1972.

[26] S. Chen, X. He, and L.-S. Luo, "Computational methods for multiphase flow," in Lattice Boltzmann Models for Multiphase Flow, A. Prosperetti and G. Tryggvason, Eds., chapter 6, pp. 157192, Cambridge University Press, Cambridge, UK, 2007. 


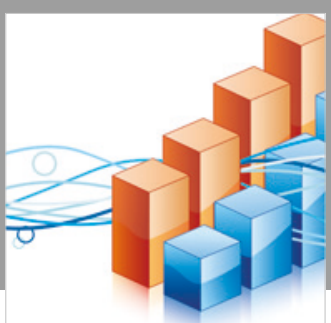

Advances in

Operations Research

\section{-n-m}
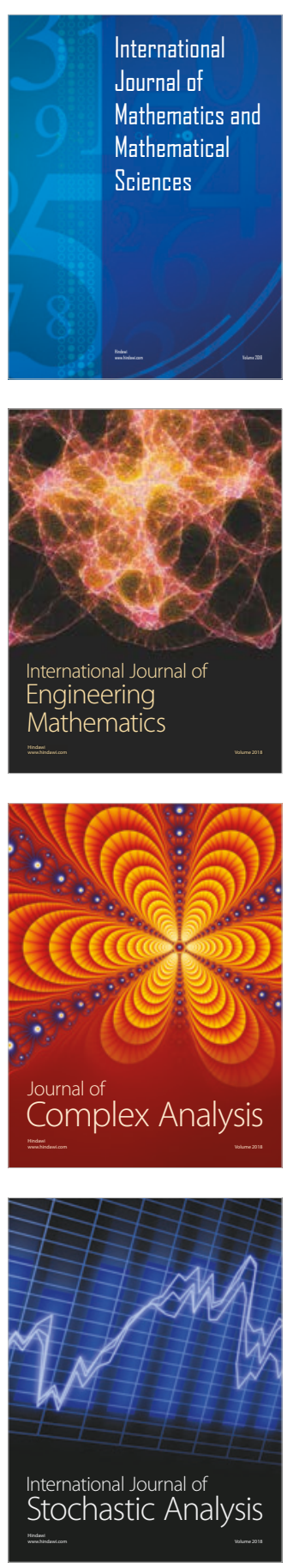
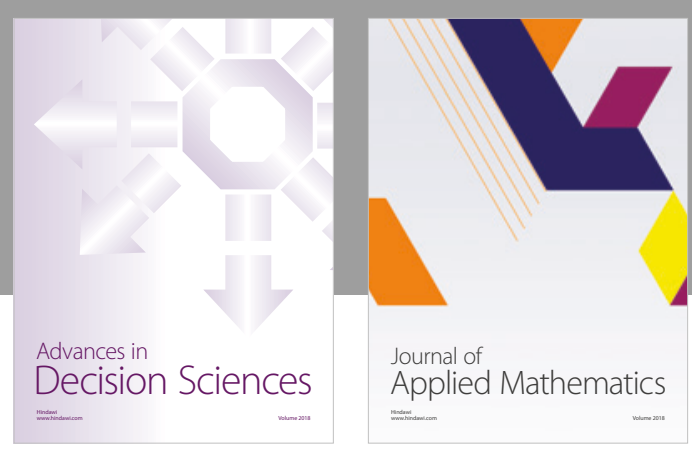

Journal of

Applied Mathematics
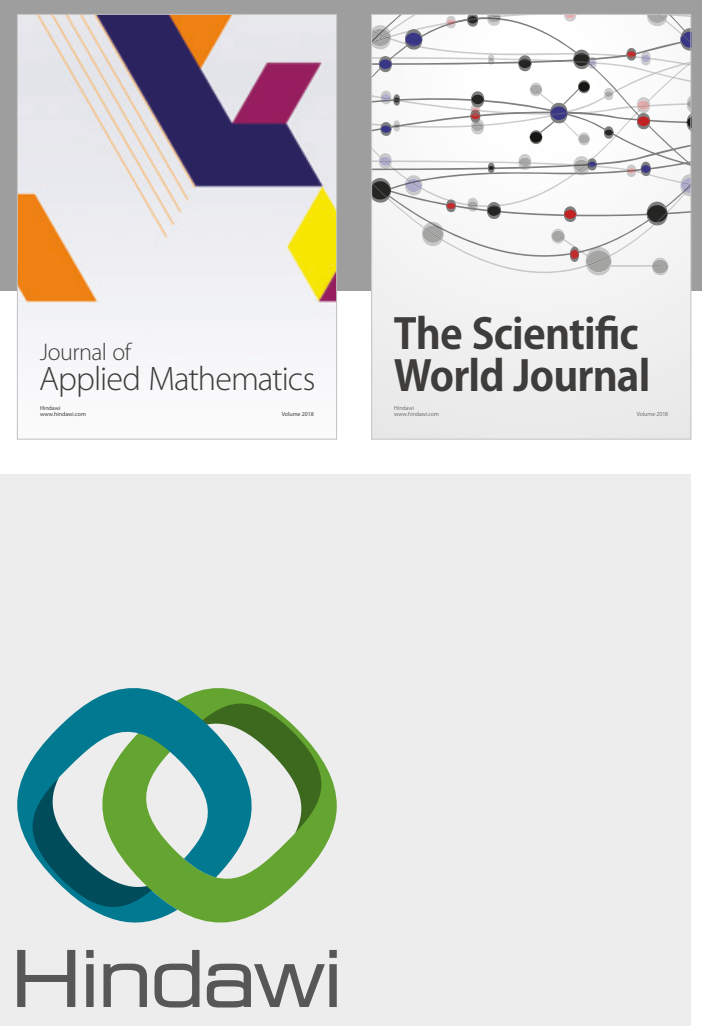

Submit your manuscripts at

www.hindawi.com

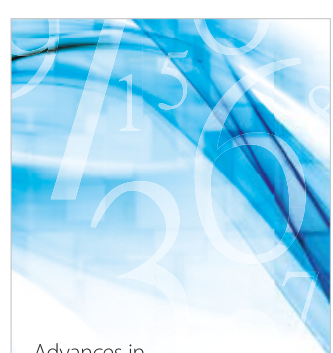

Advances in
Numerical Analysis
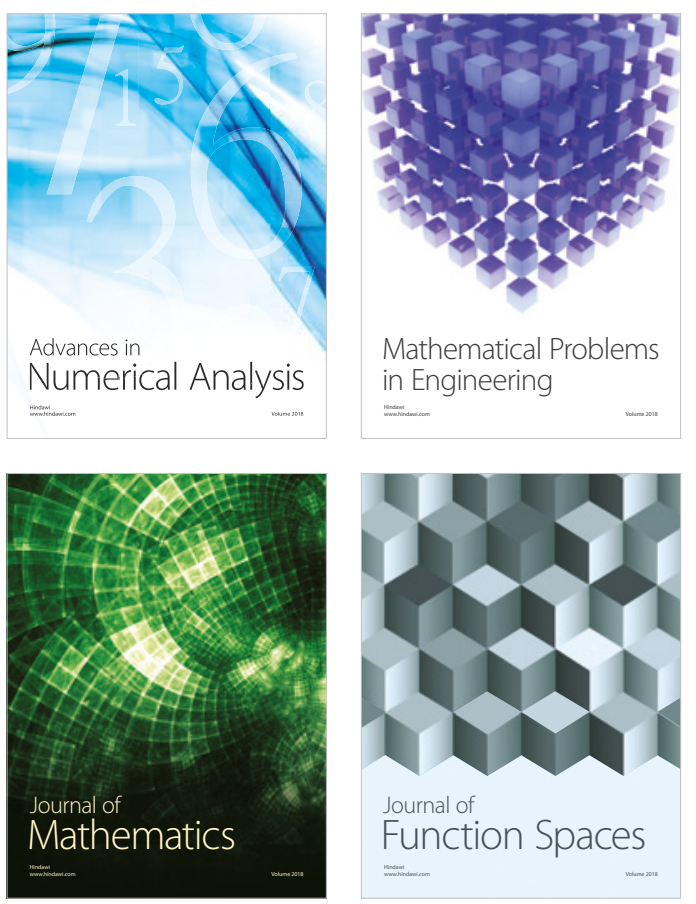

Mathematical Problems in Engineering

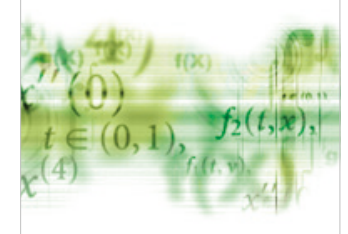

International Journal of

Differential Equations

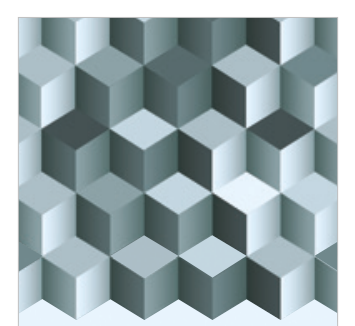

Journal of

Function Spaces

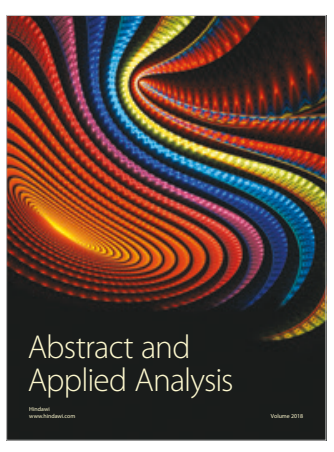

The Scientific

World Journal

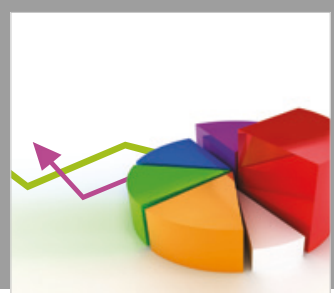

Journal of

Probability and Statistics
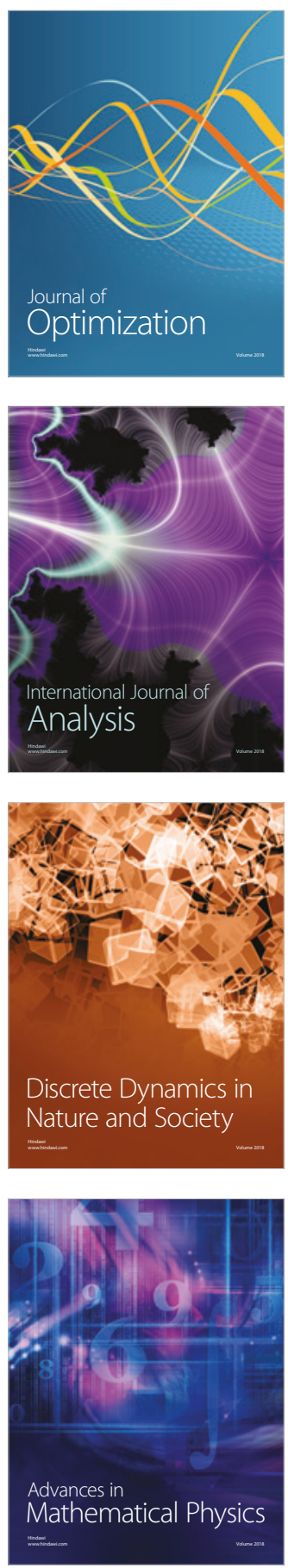